\title{
Auswirkungen möglicher Klimaänderungen auf Hochwasser und Wasserhaushaltskomponenten ausgewählter Einzugsgebiete in Österreich
}

Zusammenfassung: Der Einfluss einer möglichen Klimaänderung auf das Abflussgeschehen wurde anhand von vier österreichischen Testeinzugsgebieten - der Bregenzer Ache (Pegel Mellau), der Lavant (Pegel Fischering), der Traisen (Pegel Lilienfeld) und der Oberen Salzach (Pegel Mittersill) erforscht. Für diese Untersuchung wurden Ergebnisse (Niederschlag, Lufttemperatur) des regionalen Klimamodells REMO-UBA des deutschen Max Planck Instituts Hamburg verwendet und die Klimaänderungssignale bestimmt. Dabei fanden die Entwicklungsszenarien A1B und B1 Anwendung. Mit Hilfe eines stochastischen Wettergenerators wurden Tageswerte der Lufttemperatur und des Niederschlags generiert, wobei die Klimanormalperiode 1961-1990 als Referenzperiode und 20712100 als zukünftige Vergleichsperiode gewählt wurden. Anhand monatsweiser Analysen wurden Änderungstendenzen festgestellt und dokumentiert, wobei regional unterschiedliche Ergebnisse erzielt wurden. Generell wurden eine Niederschlagszunahme in den Wintermonaten und eine Reduktion in den Sommermonaten festgestellt. Gemeinsam mit zunehmenden Winterniederschlägen werden die Abflüsse im Winter erhöht, die Sommerabflüsse werden tendenziell geringer. An der Bregenzer Ache reduzieren sich die Hochwasserabflüsse um ca. $10 \%$, ebenso and der Oberen Salzach bei Szenario A1B. An der Lavant werden gleichbleibende bis gering ansteigende Hochwässer prognostiziert. An der Traisen wird ein deutlicher Anstieg, insbesondere bei Szenario B1 vorhergesagt. Das ereignisbezogene saisonale Auftreten von Hochwässern zeigt eine Verschiebung in Richtung kurz andauernder intensiver Regenereignisse.

The effects of potential climate changes on flood and water regime components in selected catchment areas in Austria

Summary: The effects of climate change on the runoff situation have been investigated at four Austrian catchments, Bregenzer Ache (gauge Mellau), Lavant (gauge Fischering), Traisen (gauge Lilienfeld) and Salzach (gauge Mittersill). For this analysis

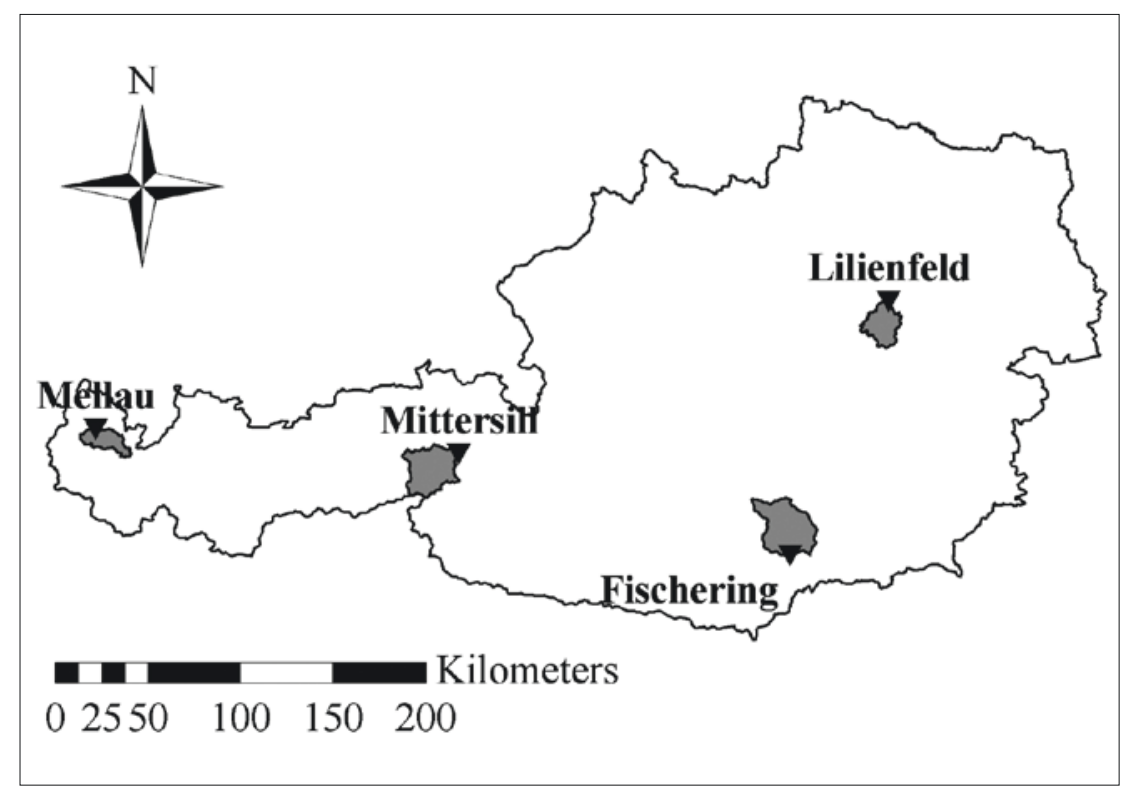

Abb. 1: Lage der Untersuchungsgebiete.

the climate change scenarios $\mathrm{A} 1 \mathrm{~B}$ and $\mathrm{B} 1$ of the regional climate model (RCM) REMO of Max-Planck-Institute-for-MeteorologyHamburg have been used for creating daily time series of air temperature and precipitation by means of a stochastic weather generator. The reference status of the period of 1961 to 1990 was compared with the period of 2070 to 2100 and the changes were interpreted as climate change impact. The results showed strong regional differences. The magnitude of the annual floods will be reduced in the alpine basins and significantly increased in the Traisen basin. An overall trend to a seasonal change in flood could be shown. The tendency of the decrease of snow accumulation and earlier snow smelt caused by higher air temperature and a higher rate in liquid precipitation with more runoff in winter time and less in summer time could be demonstrated.

\section{Einleitung}

Die Erfahrungen der großen Hochwasserereignisse in dieser Dekade (2002 Donauraum/Nordösterreich, 2005 Westösterreich und 2006 Marchfeld) wurden von der offentlichen Meinung oft als direktes Indiz für einen bereits eingetretenen Kliweis eines eindeutigen Trends beim Niederschlag aus fachlicher Sicht nicht gegeben werden kann (Böhm et al., 2008) - bei der Lufttemperatur liegt seit den Achtzigerjahren ein signifikant steigender Trend vor - ist es aus der Sicht der planenden Wasserwirtschaft durchaus gerechtfertigt, die quantitative Auswirkung möglicher Änderungen auf den Wasserhaushalt und speziell auf den Hochwasserabfluss zu erörtern. Im Rahmen des Projekts FloodriskII (BMVIT und BMLFUW, 2009) wurde es den Autoren ermöglicht, für ausgewählte Einzugsgebiete in Österreich eine Analyse hinsichtlich veränderlicher Abflussverhältnisse durchzuführen (vgl. Holzmann et. al, 2008). Besonderes Augenmerk wurde in dieser Studie der Änderung der Abflussbildung durch Schneeschmelze und den geänderten abflusswirksamen Wetter-Typen gewidmet. Die gewählte Methodik unter Anwendung eines kontinuierlichen Abflussbzw. Wasserhaushaltsmodells erlaubt aber auch eine Aussage hinsichtlich anderer Charakteristika wie Hochwasserwahrscheinlichkeit und saisonales Auftreten, mawandel interpretiert. Obwohl der Nach- 
TABELLE 1

Gebietscharakteristika und Wasserbilanz der Testeinzugsgebiete (BMLFUW, 2007).

\begin{tabular}{llllll} 
Einzugsgebiet & $\begin{array}{l}\text { Fläche } \\
{\left[\mathbf{k m}^{2}\right]}\end{array}$ & $\begin{array}{l}\text { Höhenbereich } \\
{[\mathbf{m} . \mathbf{a . s . 1}]}\end{array}$ & $\begin{array}{l}\text { Jährl. Niederschlag } \\
{[\mathbf{m m}]}\end{array}$ & $\begin{array}{l}\text { Aktuelle Evapotransp. } \\
{[\mathbf{m m}]}\end{array}$ & $\begin{array}{l}\text { Mittl. Abflusshöhe } \\
{\left[\mathbf{m m} /\left(\mathbf{m}^{3} / \mathbf{s}\right)\right]}\end{array}$ \\
\hline Bregenzer Ache & 227,5 & $600-2600$ & 2335 & 413 & $1922 /(14,2)$ \\
\hline Lavant & 714,9 & $400-2400$ & 993 & 545 & $448 /(10,2)$ \\
\hline Traisen & 331,6 & $300-1800$ & 1356 & 608 & $748 /(7,9)$ \\
\hline Obere Salzach & 590,2 & $700-3600$ & 1749 & 306 & $1443 /(27,0)$ \\
\hline
\end{tabular}

Niederwasserperioden, Schneebedeckungsdauer und Verdunstung.

In den letzten Jahren gelangten eine Vielzahl globaler (GCM) und, in diese eingebettete regionale (RCM) Klimamodelle zur Anwendung. Aufgrund unterschiedlicher Konzeptionierung, räumlicher Auflösung und Wahl der Randbedingungen liefern diese Modelle recht unterschiedliche Ergebnisse. Dies gilt besonders für die Quantifizierung der Niederschlagsentwicklung im Alpenraum. Eine Analyse der Variabilität der Modelle erfolgte u.a. im EU-Project Prudence (vgl. Cristensen et al., 2007; Frei et al., 2005). Eine weitere Variabilität in den Ergebnissen der Klimamodelle ergibt sich aus den zugrundeliegenden Emissionsszenarien der Zukunft. Dabei spielt die weltweite wirtschaftliche und gesellschaftliche Entwicklung eine große Rolle. Die berechneten Szenarien der Klimamodelle variieren zwischen uneingeschränkter Weiterentwicklung (worst case) und dem unmittelbaren Einschwenken auf Reduktionserfordernisse und sind im aktuellen IPCC Bericht (IPCC, 2007) ausführlich beschrieben.

In der zugrundeliegenden Untersuchung wurden die Szenarien B1 (optimistisch) und A1B (realistisch) berücksichtigt. Dabei fand das Regionale Klimamodell REMO-UBA vom Max Planck Institut Hamburg (Jacob et al., 2008) Anwendung. Es handelt sich dabei um ein eingebettetes (nested) Modell, das als räumliche Randbedingung die Daten des globalen Modells ECHAM5 verwendet. Die Bilanzierung der Referenzperiode (1961-1990) und der zukünftigen Periode (2071-2100) erfolgte in Eintagesschritten. Die Niederschlagsverteilung innerhalb der Testeinzugsgebiete wurde als homogen (lumped) angenommen, der Temperaturverlauf wurde in Höhenzonen zu 100 Meter unterteilt.

Die Einschränkung auf Klimadaten aus nur einem Klimamodell ergab sich aus dem vorgegebenen Projektrahmen. Dennoch können zahlreiche Ergebnisse insbesondere die Temperaturentwicklung und die saisonale Verlagerung des Niederschlages als durchaus repräsentativ ange- sehen werden. Einschränkungen ergeben sich mitunter bei der Beschreibung konvektiver Ereignisse, die einerseits im verwendeten Klimamodell nicht gut wiedergegeben werden und zum anderen daraus, dass durch die zeitliche Modellauflösung (Tageswertmodelle) subskalige Prozesse nicht hinreichend beschrieben sind.

\section{Untersuchungsgebiete}

Aufgrund der topographischen Gliederung Österreichs besteht eine regionale Variabilität der meteorologischen und klimatischen Charakteristika. Daher wurden für die durchgeführte Studie verschiedene Regionen bzw. Testeinzugsgebiete Österreichs untersucht. Es waren dies die Bregenzer Ache (Pegel Mellau), die Lavant (Pegel Fischering), die Traisen (Pegel Lilienfeld) und die Obere Salzach (Pegel Mittersill). Die Lage der Einzugsgebiete findet sich in Abbildung 1, einige Gebietskenndaten sind in Tabelle 1 dargestellt.

Das Einzugsgebiet der Bregenzer Ache ist mit $227 \mathrm{~km}^{2}$ das kleinste Testgebiet und weist den größten Gebietsniederschlag auf (2335 mm). Ca. 54 \% der Fläche sind Grünland, $39 \%$ sind bewaldet. Die Lavant hat am Pegel Fischering eine Fläche von $715 \mathrm{~km}^{2}$. Der mittlere Jahresniederschlag beträgt nur $993 \mathrm{~mm}$ und ist der geringste der Vergleichsgebiete. Die Landnutzung ist durch Nadelwälder dominiert (Flächenanteil $63 \%$ ), Grünland ist mit $31 \%$ vertreten. Die Traisen hat am Pegel Lilienfeld eine Einzugsgebietsfläche von $332 \mathrm{~km}^{2}$. Hier liegt der Wert der aktuellen Jahresverdunstung mit $608 \mathrm{~mm}$ am höchsten. Dies ist auch durch den fast $90 \%$-igen Waldanteil bedingt. Die Obere Salzach mit einer Fläche von $590 \mathrm{~km}^{2}$ hat den größten hochalpinen Flächenanteil, der zum Teil vergletschert ist (ca. $8 \%$ ). Die vegetationsarmen Flächen mit $28 \%$ haben ca. den gleichen Anteil wie Wald- bzw. Grünflächen.

\section{Methodik}

Die angewandte Methodik beinhaltet zwei thematische Schwerpunkte: Es sind dies (1) die zuverlässige Schätzung der meteorologischen Gebietskennwerte (Flächenniederschlag und Lufttemperatur) sowie (2) die Berechnung der aus dem meteorologischen Input resultierenden Abflusskomponenten. Letztere beinhaltet die hydrologischen Teilprozesse Schneeakkumulation und Schmelze, Gletscherschmelze (Obere Salzach), Abflussbildung und Abflusstransformation. Dafür gelangte in adaptierter Form ein, am Institut für Wasserwirtschaft, Hydrologie und Konstruktiven Wasserbau der Universität für Bodenkultur Wien entwickeltes Wasserbilanzmodell zur Anwendung (vgl. Holzmann und Nachtnebel, 2002).

\subsection{Meteorologie}

\subsubsection{Wahl des Klimamodells und der Entwicklungsszenarien}

Die Basis für die Erstellung der regionalen Klimaszenarien für die Einzugsgebiete bildet das globale, gekoppelte AtmosphäreOzean-Zirkulationsmodell ECHAM5/ MPI-OM vom Max Plack Institut für Meteorologie in Hamburg (Roeckner et al., 2006). Da jedoch globale Modelle aufgrund ihrer groben räumlichen Auflösung die Bedingungen im Alpenraum nicht auflösen können, wurden die dynamisch regionalisierten Klimaszenarien des regionalen Klimamodells REMO des Max Plack Institut für Meteorologie in Hamburg (Jacob et al., 2008) verwendet. Diese REMOUBA Szenarien liegen für den Kontrolllauf 1950-2000 und die Szenarienläufe 20002100 für die verschiedenen IPCC SRESSzenarien (B1, A1B, A2) vor.

Selbst die derzeit höchstaufgelösten regionalen Klimamodelle mit etwa 10 km Gitterpunktweite sind nicht in der Lage die alpinen Verhältnisse, und hier insbesondere die Niederschlagsverhältnisse mit einer Genauigkeit wieder zu geben, die eine direkte Verwendung der Modelldaten erlaubt. Die Evaluierung einiger regionaler Klimamodelle für den Winterniederschlag im Alpenraum (Krahe et al., 2008) zeigt, 
dass alle Modelle den Winterniederschlag überschätzen, wobei REMO-UBA mit etwa 65 \% Überschätzung noch relativ gut abschneidet. Neben der Niederschlagsmenge überschätzen alle Modelle aber auch die Niederschlagshäufigkeit. Aufgrund der Qualität der Absolutwerte wird klar, dass auch von den regionalen Modellen nur die Änderungen der meteorologischen Größen unter Klimaänderungsbedingungen verwendet werden dürfen. Hierzu wird die Differenz zwischen Kontrolllauf und Szenarienlauf gebildet.

Für die Analyse möglicher Änderungen wurden zwei verschiedene Klimaszenarien des REMO-UBA Modells gewählt und zwar die Emissionsszenarien B1 und A1B. B1 ist ein sehr optimistisches Szenario. Hierbei geht man davon aus, dass es gelingt bis zum Ende des Jahrhunderts die Treibhausgaskonzentrationen auf einem Niveau von $550 \mathrm{ppm}$ zu stabilisieren. Für die globale Mitteltemperatur ergibt sich bei diesem Szenario bis Ende des Jahrhunderts ein Anstieg von $1,8^{\circ} \mathrm{C}$. A1B ist ein eher realistisches Szenario. Hier geht man davon aus, dass die Treibhausgasemissionen bis in die Mitte des Jahrhunderts noch weiter stark ansteigen, danach jedoch rasch abnehmen. Innerhalb dieses Jahrhunderts kommt es noch zu keiner Stabilisierung der Treibhausgaskonzentrationen und es wird ein Niveau von mehr als 700 ppm erreicht. Dies führt zu einem globalen Temperaturanstieg von durchschnittlich $2,9^{\circ} \mathrm{C}$.

Als Zeithorizont für die lokalen Szenarien wurde das Ende dieses Jahrhundert (2071-2100) gewählt. Vor der Mitte des Jahrhunderts sind die Unterschiede zwischen den Emissionsszenarien noch sehr gering und daher ist eine Differenzierung vor der Mitte dieses Jahrhundert nicht sehr sinnvoll. Als Referenzperiode wurde die sog. Klimanormalperiode 1961-1990 herangezogen. Analysen der Änderungen beziehen sich daher auf den Vergleich dieser beiden Zeitspannen.

\subsubsection{Stochastischer Wettergenerator}

Wie bereits erwähnt überschätzen die bestehenden Klimamodelle die Absolutwerte der Niederschläge im Alpenraum signifikant. Daher ist es günstiger, die Änderungssignale zwischen berechneter Referenzperiode und Zukunftsperiode für die Modellierung heranzuziehen. Da jedoch für die Niederschlag-Abflussmodellierung kontinuierliche Zeitreihen auf Tagesbasis notwendig sind, werden diese Zeitreihen künstlich mit Hilfe eines Wettergenerators erzeugt.
Als Wettergenerator wurde der LARSWG (Long Ashton Research Station Weather Generator; Semenov und Barrow, 1997) ausgewählt und getestet. Ein Wettergenerator ist ein stochastisches Modell für die Generierung von synthetischen Wetterdaten, die der beobachteten Statistik entsprechen. Dies ist eine einfache und schnelle Methode um lange meteorologische Zeitreihen mit konstantem Klima zu erhalten. Anwendungen finden sich u.a. in Land- und Forstwirtschaftsmodellen, in der Hydrologie und allgemein in der Klimafolgenforschung. In einem ersten Schritt berechnet der Wettergenerator aus einer vorliegenden Beobachtungszeitreihe alle notwendigen statistischen Kenngrößen wie Mittelwerte, Standardabweichung und Häufigkeitsverteilungen, von Temperatur und Niederschlag, sowie die Andauer und Häufigkeit von Trockenoder Niederschlagsperioden. Umgekehrt kann der Wettergenerator nun aus diesen Kennwerten, Zeitreihen der Temperatur und des Niederschlags auf Tagesbasis berechnen, die abgesehen von stochastischen Limitierungen, genau die statistischen Kenngrößen der Beobachtung reproduzieren.

Die für den ausgewählten Standort ermittelte Statistik des beobachteten Wetters, dient im Weiteren als Grundlage zur Berechnung der Zeitreihen von Temperatur und Niederschlag für verschiedene zukünftige Klima-Szenarien.

Zur Erstellung der regionalen Klimaszenarien auf Tagesbasis, wird dem kalib- rierten Wettergenerator das Klimaänderungssignal für das jeweilige Szenario (sowohl mittlere monatliche Änderungen der Temperatur und des Niederschlages, als auch die Änderung der Länge der Trockenperioden) mitgeteilt und beliebig lange Zeitreihen für das neue Regionalklima erstellt.

Der beschriebene Wettergenerator wurde an einer Referenzklimastation im Testgebiet angeeicht. Die gewählten Referenzstationen waren Schoppernau (Bregenzer Ache), Preitenegg (Lavant), St. Ägyd im Neuwalde (Traisen) und Krimml (Obere Salzach). Dieser Stationsniederschlag wurde unter Berücksichtigung einer linearen Regressionsbeziehung an den mittels Thiessenpolygon berechneten Flächenniederschlag übergeführt. Diese Transformation zeigte für alle vier Testgebiete ein Bestimmtheitsmaß von über 0,9 . Der Sommerniederschlag lässt sich aufgrund seines hohen konvektiven (Gewitter-) Anteils am Niederschlag etwas schlechter schätzen als der Winterniederschlag.

\subsection{Hydrologische Modellierung}

Die abflussbildenden Wasserhaushaltskomponenten sind der Niederschlag in Form von Regen und der Schmelzanteil aus Schnee und Gletscherflächen. Diese bilden den Modellinput für die hydrologische Modellierung, die mit Hilfe eines am IWHW der Univ. f. BOKU entwickelten Abflussmodells erfolgte. Eine Schemenskizze dazu findet sich in Abbildung 2.

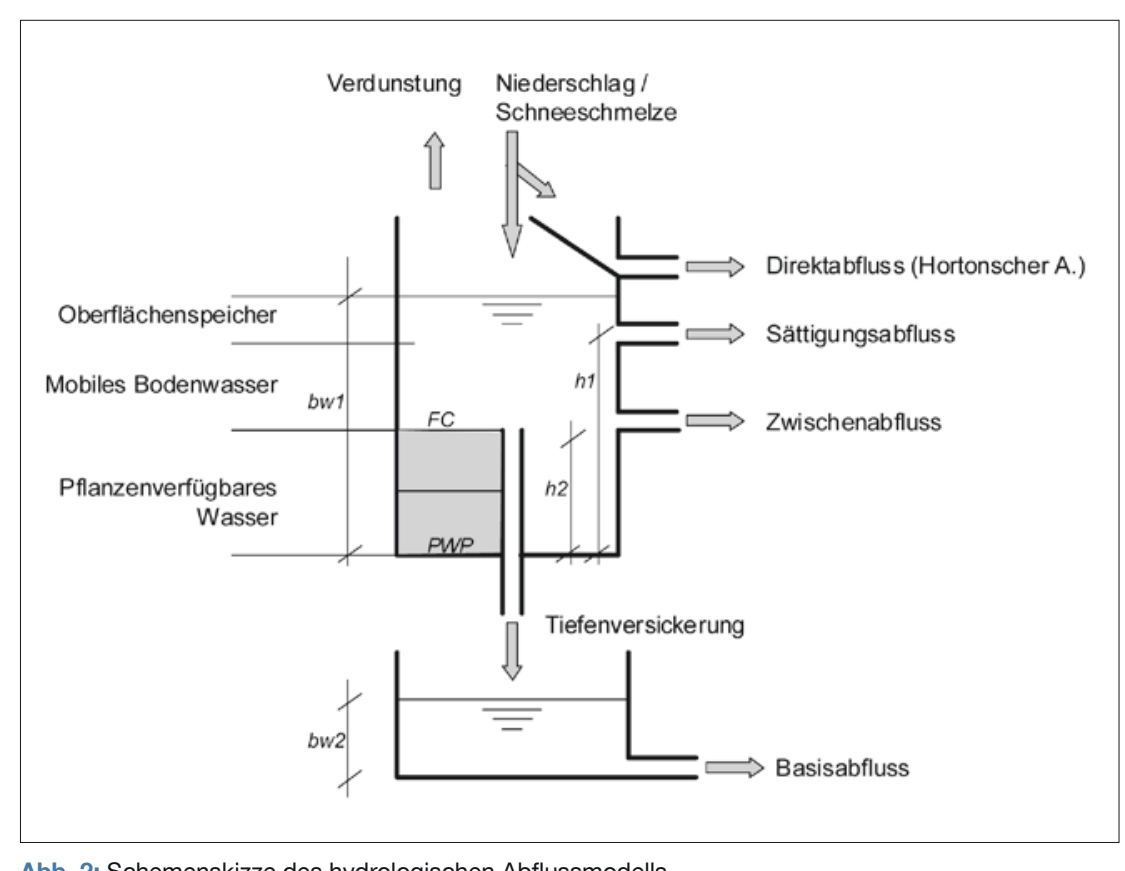

Abb. 2: Schemenskizze des hydrologischen Abflussmodells. 


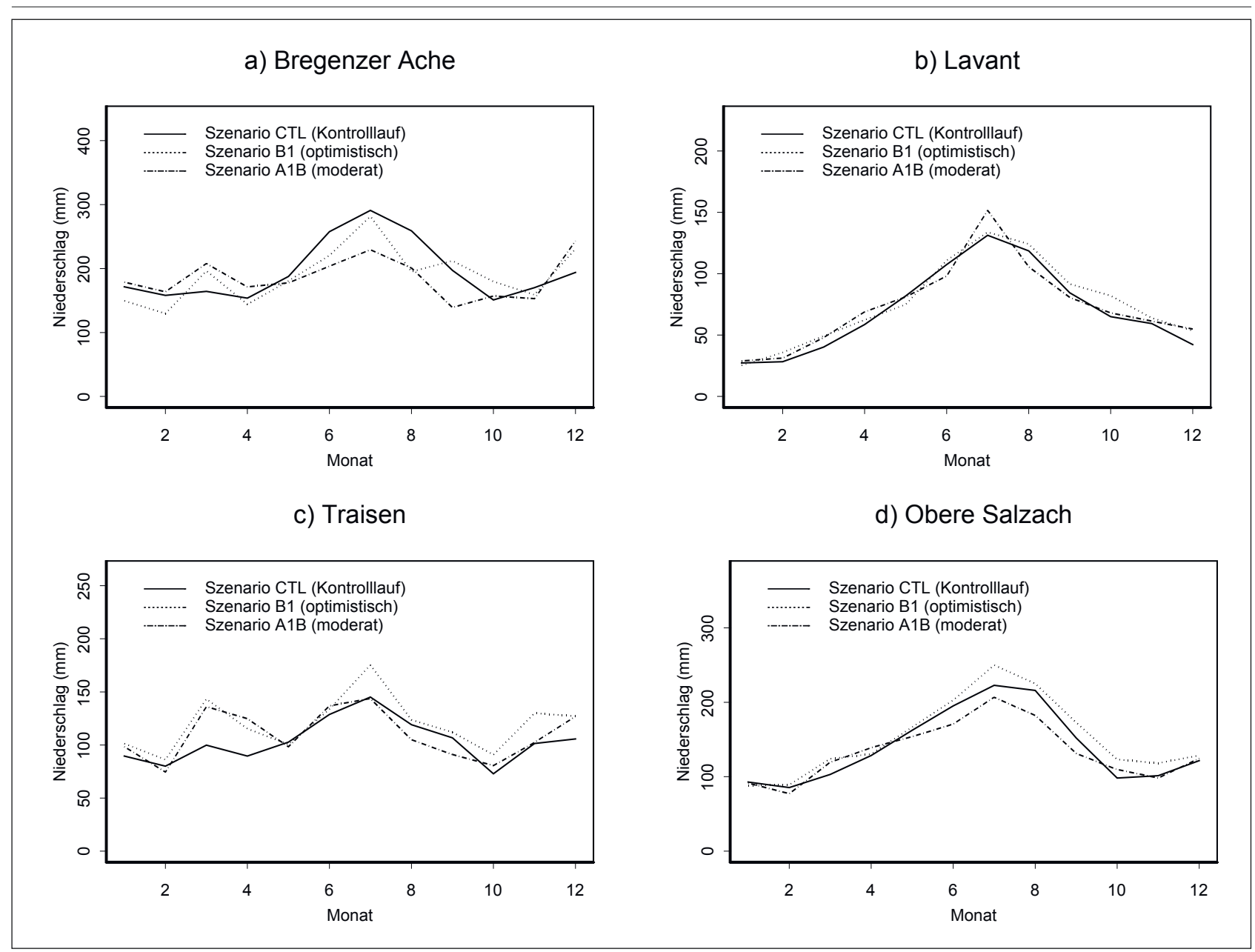

Abb. 3: Mittlere Monatsniederschläge für die Referenzperiode und den Prognosezeitraum in den Testgebieten Bregenzer Ache (a), Lavant (b), Traisen (c) und Obere Salzach (d).

Das Modell berücksichtigt unterschiedliche Abflusskomponenten wie Oberflächenabfluss (Hortonscher Abfluss), Sättigungsabfluss, Zwischenabfluss und Basisabfluss (Grundwasserzustrom), deren Summe dem Gesamtabfluss am Gebietsauslass entspricht. Der Systemzustand wird durch den Füllungsgrad des Bodenwasserspeichers (bw1) und dem Grundwasserspeicher (bw2) definiert. Aus dem Bodenwasserspeicher erfolgt auch die Verdunstung (Evapotranspiration), deren potentieller Wert nach der Formel von Thornthwhaite berechnet wird. Die aktuelle Verdunstung (reduzierte Verdunstung) wird bei Unterschreitung des Bodenwasserwertes unter 1/2 h2 wirksam.

Niederschlag und Schneeschmelze infiltrieren zum Teil in den Bodenspeicher, ein Anteil trägt jedoch über den Direktabflussspeicher unmittelbar zum Abfluss bei. Dies berücksichtigt die begrenzte Infiltrationskapazität des Bodens bei Starkregen bzw. bei gefrorenem Untergrund.
Im Falle einer Schneeakkumulation reduziert sich der wirksame Niederschlag das ist jener Niederschlag, der in Form von Regen fällt - gemäß dem Flächenanteil jener Zonen, in denen Schneefall auftritt.

Die Schneeschmelze wird mittels Temperaturindexverfahren (Grad-Tag-Verfahren) berechnet. Dabei erfolgt eine höhenbezogene Differenzierung der Lufttemperatur durch Untergliederung in 100-Meter Höhenzonen mit Hilfe eines feuchtadiabatischen Gradienten von $-0,65{ }^{\circ} \mathrm{C} / 100 \mathrm{~m}$. Die Berechnung der Gletscherschmelze verwendet einen vergleichbaren Ansatz unter der Voraussetzung einer Ausaperung der überlagernden Schneedecke in dieser Zone. Der Gletscherabfluss wird über einen Linearspeicher direkt zum Gebietsauslass transferiert. Sämtliche Modellparameter wurden anhand beobachteter Datenreihen von 1971 bis 2004 kalibriert und validiert. Dabei wurde der Gebietsniederschlag nach der o.g. Methode aus nur einer Referenzstation im Gebiet ermittelt. Das Bestimmt- heitsmaß lag zwischen 0,55 (Traisen) und 0,73 (Obere Salzach).

\section{Ergebnisse}

Die untersuchten Einzugsgebiete zeigen je nach ihrer geographischen und orographischen Lage doch ein sehr unterschiedliches Verhalten. Diese Differenzierung wird hier unter Bezugnahme auf die lokalen Klimabedingungen und deren Auswirkungen auf das Hochwassergeschehen, die mittleren Abflussverhältnisse, Niederwasser sowie auf Schneebedeckungsverhältnisse zusammengefasst. Die in Betracht gezogenen Klimaszenarien sind Szenario B1 (optimistisch) und Szenario A1B (realistisch). Der Änderungszeitraum beschreibt die Verhältnisse 2071-2100 im Vergleich zur Referenzperiode 1961-1990.

\subsection{Meteorologische Entwicklung}

Grundsätzlich ist bei beiden Klimaszenarien mit einer Erhöhung der Lufttempera- 


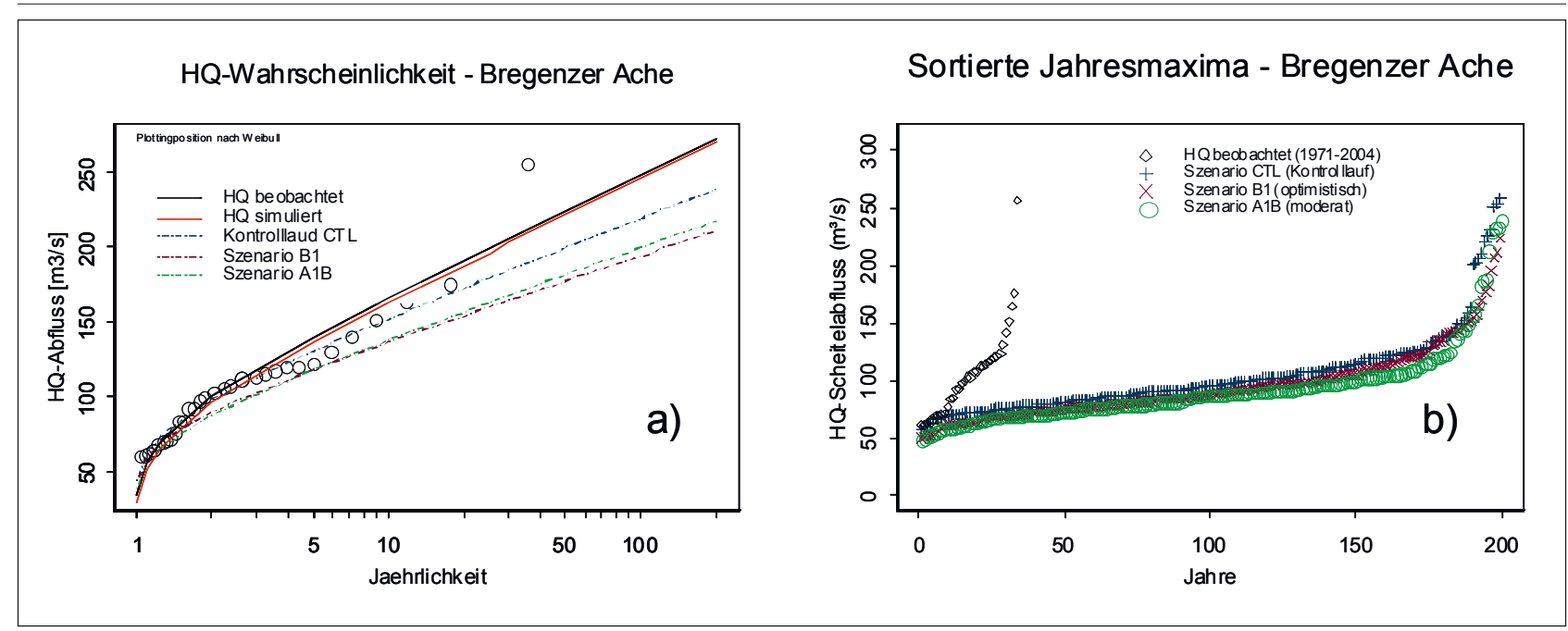

Abb. 4: Extremwertverteilung nach Gumbel (a) und beobachtete und simulierte Jahreshochwässer (b).

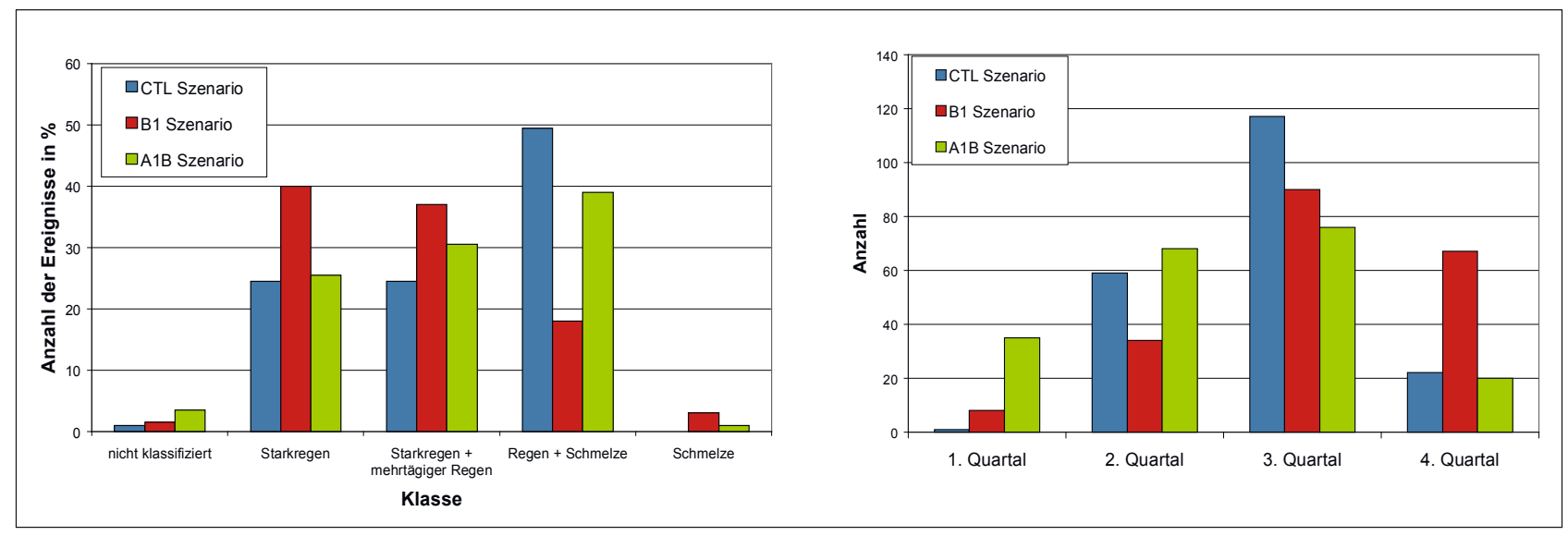

Abb. 5: Hochwassererzeugende Niederschlagstypen (a) und deren saisonales Auftreten (b) für die Bregenzer Ache.

tur zu rechnen. Im Nordosten Österreichs ist der mittlere Temperaturanstieg moderater $\left(2,5-3,0^{\circ} \mathrm{C}\right)$, im Westen und Süden werden Anstiege bis $4{ }^{\circ} \mathrm{C}$ erwartet. Szenario A1B liefert etwa $1,0-1,5^{\circ} \mathrm{C}$ höhere Temperaturanstiege als Szenario B1.

Die Bregenzer Ache weist tendenziell einen Rückgang des Niederschlags aus wobei jedoch eine Intensitätszunahme im Winter bei Szenario A1B auftritt. Die Anzahl der Regentage im Sommer nimmt ab. Bei der Lavant sind die erwarteten Änderungen in den Monatsniederschlägen gering. An der Traisen kann man einen deutlichen Anstieg der Frühjahresniederschläge erkennen. Sommer- und Herbstniederschläge steigen bei Szenario B1. Bei der Oberen Salzach besteht ein ausgeprägter Unterschied zwischen A1B und B1. Szenario B1 liefert fast für den gesamten Jahresverlauf höhere Niederschläge, A1B zeigt geringere Niederschläge während der Sommermonate (vgl. Abb. 3).

\subsection{Hochwasser und Abflusstypen}

Die Ergebnisse basieren auf den Jahresabflussmaxima (Tageswerte) von 200-jährigen, mittels Wettergenerator erzeugten $\mathrm{Si}$ mulationsperioden, welche die Klimaverhältnisse des Referenzzeitraums 1961-1990 bzw. des Prognosezeitraums 2071-2100 umfassen. Am Beispiel der Bregenzer Ache sind die sortierten Jahrshochwässer (siehe Abb. 4b) und die resultierende Extremwertverteilung nach Gumbel (siehe Abb. 4a) dargestellt.

Das extreme Ereignis vom 22.5.1999 (HQ-Scheitel $257 \mathrm{~m}^{3} / \mathrm{s}$ ) führt zu einer deutlichen Anhebung der statistischen HQ-Werte (vergl. dazu Tab. 2). Diese Problematik der HQ-Ausreisser wurde auch in Blöschl et al. (2008) beschrieben. Die Extremwerte der 200-jähigen Simulationsperioden entsprechen ebenfalls ca. diesem Wert. Aus dem Vergleich der Szenarien und dem Kontrolllauf leiten sich Interpretationsmöglichkeiten hinsichtlich der erwarteten Änderungen der HQ-Werte für die Zukunft ab. Diese Vergleichswerte sind in Tabelle 3 dargestellt. Es ergeben sich für die Bregenzer Ache kleinere HQWerte bei beiden Szenarien. Die maximalen Verringerungen sind grün hinterlegt, für den HQ100-Wert wird eine Reduktion um $11.5 \%$ errechnet. An der Oberen Salzach wird bei Szenario A1B ebenfalls eine Verringerung der HQ-Werte erwartet, bei Szenario B1 eine Erhöhung um ca. 7 \%. An der Lavant wird bei Szenario B1 eine moderate Erhöhung der HQ-Werte um ca. 6 \% erwartet, bei Szenario A1B bleiben die Verhältnisse unverändert. An der Traisen wurde eine signifikante Erhöhung bis zu $34 \%$ errechnet. Dies trifft besonders bei den rot hinterlegten Werten des Szenario B1 zu, bei Al erfolgt eine Erhöhung um ca. $10 \%$. Die relativ großen Schwankungsbereiche der Änderungen decken sich auch mit den Ergebnissen von Blöschl et al. (2008) in denen für die Untersuchungsgebiete Gail und Pielach je nach Szenario 


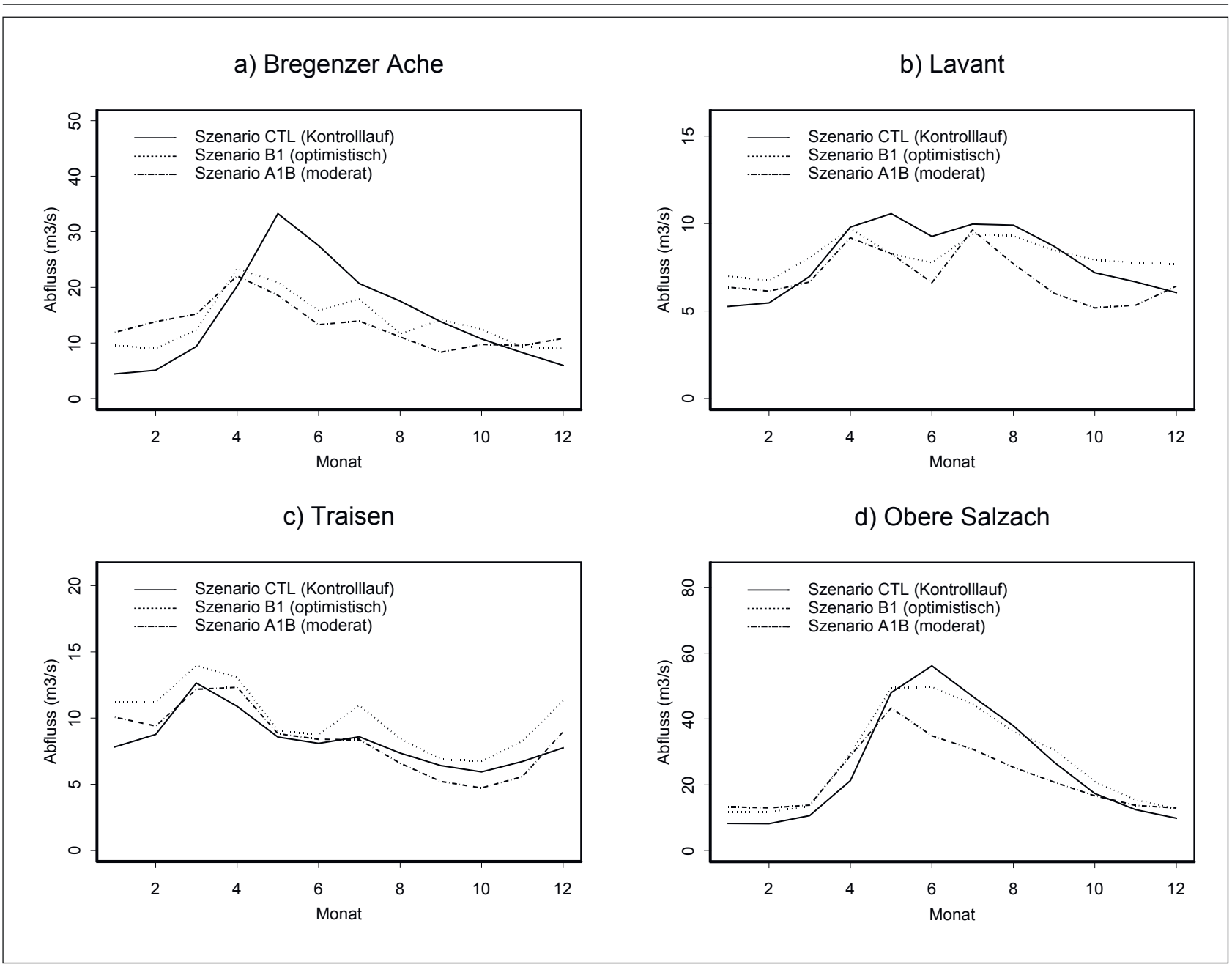

Abb. 6: Mittlerer Abfluss für die Referenzperiode und den Prognosezeitraum in den Testgebieten Bregenzer Ache (a), Lavant (b), Traisen (c) und Obere Salzach (d).

Änderungen der HQ100-Werte von $\pm 10 \%$ ausgewiesen werden. Die extremen Änderungen an der Traisen sind u.a. auch durch den Anstieg der mittleren Hochwässer und der daraus resultierenden Verschiebung der Verteilungsfunktion verbunden. Die absoluten Extremwerte unterscheiden sich zwischen Kontrolllauf und Szenarien nur geringfügig. Dies verweist einmal mehr auf die Problematik der Extremwertstatistik und der daraus abgeleiteten Bemessungsgrößen.

Weiters wurden Analysen hinsichtlich des saisonalen Auftretens von Hochwässern und dem zugrundeliegenden Niederschlagstyp durchgeführt und für die Bregenzer Ache graphisch dargestellt (vlg. $A b b .5)$. Die für den Referenzzustand häufigste Ereignisklasse (50 \%) ist das kombinierte Ereignis Regen mit Schneeschmelze. Dieser Typus reduziert sich bei Szenario B1 auf $18 \%$, bei A1B auf $39 \%$. Bei Szenario B1 bilden eintägige Starkregenereignisse die häufigste Klasse (40 \%), aber auch Star-

\begin{tabular}{llllll}
$\begin{array}{l}\text { TABELLE } 2 \\
\text { Beobachtete und simulierte HQ-Jährlichkeiten an der Bregenzer Ache } \\
\text { (Mellau). }\end{array}$ & $\mathbf{2}$ & $\mathbf{5}$ & $\mathbf{1 0}$ & $\mathbf{3 0}$ & $\mathbf{1 0 0}$ \\
\hline Jährlichkeit & 99.9 & 139.6 & 165.8 & 205.5 & 248.0 \\
\hline Beobachtet (1971-2004) & 96.8 & 126.9 & 146.8 & 176.8 & 209.0 \\
\hline Beobachtet (1971-2004 ohne 1999) & 96.1 & 136.2 & 162.7 & 202.8 & 245.7 \\
\hline Simuliert (1971-2004) & 98.1 & 130.5 & 152.0 & 184.5 & 219.3 \\
\hline Kontroll-Lauf CTL & 90.2 & 118.0 & 136.3 & 164.2 & 194.1 \\
\hline Szenario B1 & 88.0 & 117.8 & 137.6 & 167.5 & 199.6 \\
\hline Szenario A1B & & & & &
\end{tabular}

kregen über mehrere Tage tritt deutlich öfter auf als im Referenzzustand. Der häufigste Auftrittszeitraum ist im Ist-Zustand das 3. Quartal, bei B1 kommt es auch zu einem Anstieg des Auftretens im 4. Quartal, bei Szenario A1B gibt es eine Zunahme der Häufigkeiten im 1. und 2. Quartal.

An der Lavant ist der dominante Ereignistypus Starkregen. Dieser steigt von $60 \%$ auf $73 \%$ (bei B1) und auf $69 \%$ bei A1B.
Das häufigste Auftreten erfolgt im Referenzzustand und bei den Szenarien im 3. Quartal. Für Szenario B1 gibt es auch eine Häufigkeitssteigerung im 4. Quartal. Die größte Anzahl der Jahreshochwässer an der Traisen wird von Starkregenereignissen gebildet, die Änderungen im Ereignistyp aufgrund der Szenarien sind nicht gravierend. Das häufigste Auftreten erfolgt für alle Szenarien im 3. Quartal. Die Größe 


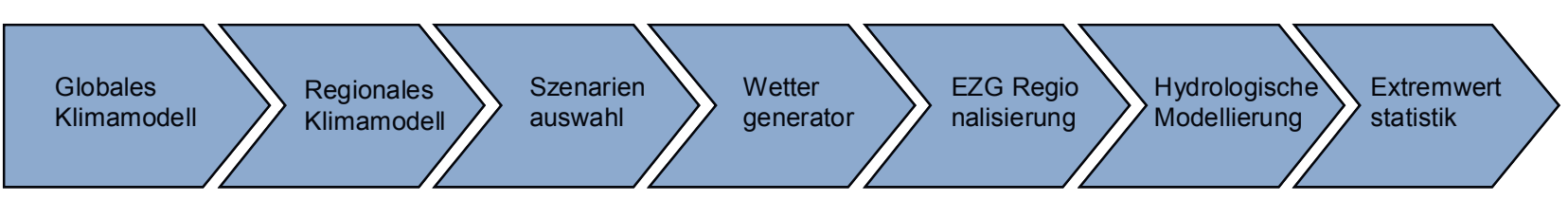

Abb. 7: Modellkette der verwendeten Daten und Verfahren.

der Jahreshochwässer nimmt an der Oberen Salzach für das Szenario A1B ab, für B1 geringfügig zu. Der bislang dominierende Ereignistyp Regen auf Schnee nimmt bei beiden Szenarien gegenüber dem Referenzzustand von $70 \%$ auf ca. $36 \%$ ab. Dafür erhöhen sich die Klassenhäufigkeiten für Starkregen sowie mehrtägige Starkregen. Das häufigste Auftreten erfolgt im 3. Quartal. Bei Szenario A1B ist eine zunehmende Häufung im 2. Quartal zu beobachten.

\subsection{Mittlere Abflussverhältnisse}

Für sämtliche Testgebiete kann ein generelles Muster beobachtet werden. Es ist dies die Abflusszunahme in den Wintermonaten bedingt durch höhere Winterniederschläge, die zum Teil aufgrund der erhöhten Temperaturen als Regen abflusswirksam werden. Des Weiteren ergibt sich eine Abflussreduktion im Herbst beim niederschlagsärmeren Szenario A1B (vgl. Abb. 6). Im Detail ergeben sich die Änderungen wie folgt: Die Bregenzer Ache zeigt eine deutliche Abflussverminderung bei beiden Szenarien in den Monaten Mai bis August und eine Erhöhung zwischen Dezember und März. Auch an der Lavant liefern die Szenarien für Mai und Juni geringere mittlere Abflüsse. Auch die deutliche
Abflussreduktion bei Szenario A1B während der Herbstmonate (3. Quartal) ist offensichtlich. An der Traisen liefert Szenario B1 durchwegs höhere mittlere Abflüsse als der Referenzzustand. Szenario A1B zeigt geringere Abflüsse in den Herbstmonaten. An der Oberen Salzach weist das Szenario AlB speziell zwischen Juni und September deutlich geringere Abflüsse auf. Das Wegfallen des Gletscherabflusses bewirkt eine deutliche Reduktion der mittleren Abflüsse in diesen Monaten.

\subsection{Niederwasser}

Niederwasser - quantifiziert anhand des Q95-Wertes - tritt an der Bregenzer Ache für den Referenzzeitraum fallweise in den Monaten Jänner bis März auf. Bei den Szenarien verschiebt sich der Auftrittszeitraum in den Herbst, wobei bei A1B mit häufigerem Auftreten zu rechnen ist. An der Lavant traten bislang Niederwasserphasen im Herbst auf. Bei beiden Klimaänderungsszenarien wird diese Häufigkeit verstärkt, wobei bei A1B das Maximum im Oktober mit ca. 40 \% Häufigkeit zu erwarten ist. Ein ähnliches Bild ergibt sich an der Traisen. Die Herbstniederwasserphasen sind bei Szenario B1 ähnlich wie im Referenzzustand, bei A1B nehmen sie jedoch deutlich zu und errei-

TABELLE 3

HQ-Änderungen der Szenarien gegenüber dem Kontrolllauf (in Prozent).

\section{HQ}

\begin{tabular}{lrrrrr}
\hline Bregenzer Ache: & $\mathbf{2}$ & $\mathbf{5}$ & $\mathbf{1 0}$ & $\mathbf{3 0}$ & $\mathbf{1 0 0}$ \\
\hline Szenario B1 & -8.1 & -9.6 & -10.3 & -11.0 & -11.5 \\
\hline Szenario A1B & -10.3 & -9.7 & -9.5 & -9.2 & -9.0 \\
\hline Lavant: & & & & & \\
\hline Szenario B1 & 4.7 & 5.5 & 5.8 & 6.2 & 6.5 \\
\hline Szenario A1B & 3.2 & 1.6 & 0.8 & 0.0 & -0.6 \\
\hline Traisen: & & & & & \\
\hline Szenario B1 & 27.2 & 30.6 & 32.1 & 33.5 & 34.6 \\
\hline Szenario A1B & 15.4 & 13.0 & 12.0 & 10.9 & 10.2 \\
\hline Obere Salzach: & & & & & \\
\hline Szenario B1 & 8.9 & 8.0 & 7.6 & 7.1 & 6.7 \\
\hline Szenario A1B & -9.3 & -7.7 & -6.9 & -5.9 & -5.2 \\
\hline
\end{tabular}

chen ca. $45 \%$ im Oktober. An der Oberen Salzach traten Niederwasserphasen meist zwischen Jänner und März auf. Bei Szenario B1 treten kaum mehr Niederwasser auf, bei A1B fallweise während der Herbstmonate.

\subsection{Schneebedeckung bzw. gespeichertes Wasseräquivalent}

Der Schneewasserwert verringert sich infolge der reduzierten Schneemengen bei allen Einzugsgebieten. In den niedriger gelegenen Gebieten Traisen und Lavant reduziert sich die in der Schneedecke gespeicherte Wassermenge um mehr als $60 \%$ bei Szenario B1 und $80 \%$ bei Szenario A1B. Die stärkere Reduktion bei Szenario A1B zeigt sich auch in den alpinen Einzugsgebieten der Bregenzer Ache und der Oberen Salzach.

\section{Ausblick und Empfehlungen}

Die hier vorgestellte Methodik verwendet Klimaänderungssignale eines eingebetteten regionalen Klimamodells. Die meteorologischen Gebietsdaten werden mit Hilfe eines stochastischen Wettergenerators erzeugt, die als Eingangsdaten für ein hydrologisches Wasserhaushaltsmodell dienen. Demgemäß ist die Modellkette (vgl. Abb. 7) äußerst komplex und die damit verbundenen Unsicherheiten sind groß.

Dennoch liefern die hier vorgestellten Analysen gut vergleichbare Ergebnisse mit anderen zentraleuropäischen Studien wie z.B. für Süddeutschland (KLIWA, 2007) oder der Schweiz (KOHS, 2007).

Auch bei anderen methodischen Ansätzen wie z. B. bei Nachtnebel (2008) in denen statistische Downskalingverfahren anhand von Wetter-Typen vorgestellt werden oder bei Blöschl et al. (2008) wo meteorologische Inputdaten gemäß den erwarteten Änderungen aus der Literatur für hydrologische Abflussmodellierung verwendet wurden, sind die grundsätzlichen Aussagen gleich. Sie werden wie folgt zusammengefasst:

- Die Lufttemperatur wird je nach Szenario um ca. 2 bis $4^{\circ} \mathrm{C}$ höher.

- Der Niederschlag nimmt im Winter (verstärkt auch als Regen) zu. Sommer- 
niederschläge werden geringer, jedoch z. T. mit höheren Intensitäten.

- Es kommt zu einer saisonalen Verschiebung der mittleren Abflüsse in den alpinen Gebieten. Niederwässer werden im Winter seltener, im Herbst häufiger.

- Die Hochwasserentwicklung ist regional unterschiedlich und stark von den Szenarien bestimmt. Es besteht ein regionaler Trend von leichter Zunahme im Nordosten zu leichter Abnahme im Westen Österreichs.

Nachfolgend werden aus den Erkenntnissen der Studien des Workpackages Hydrologie des Floodrisk II Projekts die wesentlichen abgeleitete Empfehlungen aufgelistet:

- Die Variabilität der Klimaszenarien lässt aufgrund der unterschiedlichen Ergebnisse keine eindeutige Vorgangsweise zu. Vorab müsste eine Festlegung auf ein wahrscheinliches Szenario (evtl. A1B) erfolgen.

- Eine einheitlich österreichweite Strategie (z. B. Erhöhung schutzwasserbaulicher Dimensionierungen) ist aufgrund der regionalen Unterschiede NICHT zielführend.
- Die Festlegung aktualisierter Hochwasserkennwerte sollte neben der Anwendung der regulären Extremwertstatistik auch unter Berücksichtigung von Hochwasserdekaden und historischer Extremwerte, (vgl. auch Blöschl et al, 2008) bzw. durch kontinuierliche Beurteilung neuer Ereignisse erfolgen (vgl. KOHS, 2007).

- Die Aussagen hinsichtlich der Auswirkungen des Klimawandels auf die Hochwasserentwicklung sind aufgrund des Zusammenspiels der komplexen Einflussfaktoren (vgl. Modellkette $A b b .7)$ sehr unsicher. Die Weiterentwicklungen in der Klimamodellierung und aktuelle weltweite methodischen Studien lassen eine Verbesserung der Aussageschärfe innerhalb der nächsten Dekade erwarten. Als wesentlicher Unsicherheitsfaktor verbleibt jedoch die Festlegung und Umsetzung der globalen Klimastrategien.

\section{Danksagung}

Die Autoren bedanken sich beim Bundesministerium für Land- und Forstwirt- schaft, Umwelt und Wasserwirtschaft für die Beauftragung des Teilprojekts TP1.2. im Rahmen des Schwerpunkts Floodrisk II. Weiterer Dank gilt der Zentralanstalt für Meteorologie und Geodynamik und der Abteilung Wasserwirtschaft des Lebensministeriums für die Bereitstellung der hydrographischen und meteorologischen Daten. Dem Max-Planck Institut für Meteorologie in Hamburg und dem Umweltbundesamt Deutschland danken wir für die Bereitstellung der REMO-UBA Szenarienläufe. Weiters danken wir dem Umweltbundesamt Österreich, mit dem gemeinsam die großen Datenmengen von Deutschland nach Österreich transferiert wurden.

\section{Korrespondez:}

\section{Ao. Univ.-Prof. DI Dr. Hubert Holzmann}

Institut für Wasserwirtschaft, Hydrologie und Konstruktiven

Wasserbau
Universität für Bodenkultur Wien

Muthgasse 18

Muthgasse
1190 Wien

E-Mail: hubert.holzmann@boku.ac.at.

Institut für Wasserwirtschaft, Hydrologie und Konstruktiven Wasserbau, Universität für Bodenkultur Wien, Muthgasse 18, 1190 Wien

Institut für Meteorologie, Universität für Bodenkultur Wien, Peter Jordan Str 92, 1190 Wien

\section{LITERATUR}

Blöschl G, Viglione A, Heindl H (2008) Dynamik von Hochwasserbemessungsgrößen und Konsequenzen - Klimaänderung. Endbericht zum Teilprojekt TP6.2 von Floodrisk II. Bericht an den Auftraggeber BMLFUW.

Auftraggeber BMLFUW. terreichs. 3. Lieferung. Wien: Bundesministerium für Land- und Forstwirtschaft, Umwelt und Wasserwirtschaft. ISBN 3-85437-250-7.

BMVIT und BMLFUW (2009) FloodRisk II - Vertiefung und Vernetzung zukunftsweisender Umsetzungsstrategien zum integrierten Hochwassermanagement. Synthesebericht, ISBN 978-3-85174-071-4

BÖHM R, Godina R, Nachtnebel H, Pirker O, (2008) Mögliche Klimafolgen für die Wasserwirtschaft in Osterreich. In: Auswirkungen des Klie Wasserwirtschaft. Hrsg. Von BmLFUW und ÖWAV, Wien, $7-26$

Christensen JH, Christensen OB (2007) A summary of the PRUDENCE model projections of changes in European climate by the end of this century. Clim Change, Vol. 81, 7-30.

Frei C, Schöll R, Fukutome S, Schmidli J, Vidale PL (2005) Future Change of Precipitation
Extremes in Europe: An Intercomparison of Scenarios from Regional Climate Models, J. Geophys. Res., Res. III, Art. No D06105 Holzmann H, Nachtnebel HP (2002) Abflussprognose für mittelgroße Einzugsgebiete - Methodik und Anwendungen. Österr. Wasser- und Abfallwirtschaft, 54, 9-10, 142-153.

Holzmann H, Lehmann Th, Haas P, Formayer H (2008) Auswirkung möglicher Änderungen der Schnee-, Regenanteile in Hinblick auf die Ab-

flussbildung bei Klimaänderung. Endbericht zum Teilprojekt TP1.2 von Floodrisk II. Bericht an den Auftraggeber BMLFUW.

IPCC (2007) Climate Change 2007 - The Physical Science Basis: Working Group I Contribution to the Fourth Assessment Report of the IPCC. Cambridge University Press. ISBN-13: 978 0521705967

Jacob D, Göttel H, Kotlarski S, Lorenz P,

Sieck K (2008) Klimaauswirkungen und Anpassung in Deutschland - Phase 1: Erstellung regionaler Klimaszenarien für Deutschland. ISSN 1862-4359

KLIWA (2007) Klimawandel und Konsequenzen für die Wasserwirtschaft: KLIWA-Hefte Nr. 1-12. www.kliwa.de.
KOHS (2007) Auswirkungen der Klimaänderung auf den Hochwasserschutz in der Schweiz - ein Standortpapier der Kommission Hochwasserschutz im Schweizerischen Wasserwirtschaftsverband (KOHS). Wasser-Energie-Luft, Heft $1 / 2007$.

Krahe K, Korck J, Tomassini L, Gelhardt U, Smiatek G, Formayer H (2008) Global and regional climate projections for the Alpine space. In: port ClimChAlp Interreg III B Alpine Space, Work Pakage (5): 47-88.

Nachtnebel HP (2008) Auswirkungen von möglichen Klimaänderungen auf die Hydrologie und Wasserwirtschaft in einigen österreichischen Regionen. In: Auswirkungen des Klimawandels auf die österreichische Wasserwirtschaft. Hrsg. Von BmLFUW und ÖWAV, Wien, 27-52.

Roeckner E, Brasseur G, Giorgetta M, Jaco D, Jungclaus J, Reick Ch, Sillmann J (2006) Klimaprojektionen für das 21. Jahrhundert. Mitteilung des MPI für Meteorologie Hamburg Semenov M, Barrow E (1997) Use of a stochastic weather generator in the development of climate change scenarios. Climate Change, 35 397-414 